\title{
MULTILEVEL MODELING IN HETEROGENEOUS WIRELESS SENSOR NETWORK FOR IMPROVED ENERGY EFFICIENCY
}

\author{
R. Lakshminarayanan and S. Sakthivel \\ Department of Computer Science and Engineering, Gnanamani College Technology, India
}

\begin{abstract}
In this paper, two primary and secondary parameters are proposed for a heterogeneous network model (HNM). In such a model, the heterogeneous network describes nodes with a finite energy level based on the parameter value. It evaluates the performance of the proposed HNM protocol and further tests the HNM multilevel protocol. For a finite heterogeneity level MHNM protocol is denoted by MLHNM-n. The secondary parameter determines the total nodes at each level of heterogeneity. The proposed protocol provides two parameters for determining the energy and density of the cluster heads of residual nodes. The proposed protocol will be tested by six level networks. The energy dissipation decreases and results in an increase in network life.
\end{abstract}

\section{Keywords:}

Network Lifetime, Multi Heterogeneity, Number of Rounds, Clustering

\section{INTRODUCTION}

Progress in wireless communication has made it possible to design Wireless Sensor Networks, containing tens or miles of small nodes and can be used in various circumstances as a powerful tool to collect data. The energy dissipation decreases and results in an increase in network life. A WSN is a self organized collection of sensor nodes that can be communicated via wireless medium [1]. These small sensing devices include data storage memory, CPUs for data processing, batteries and transceivers for data receipt and transfer. The sensor nodes in WSNs are reserved for low wireless bandwidth communication, low processing capacity, small storage and an inadequate battery which cannot be recharged [2].

The size of each node varies depending on application requirements, i.e. it may be microscopically decreased in military or monitoring applications. The expense depends on the processing speed, storage, battery size etc. The costs of these devices. There are two classes of WSNs: homogeneous and heterogeneous networks.

All sensor nodes are identical in hardware and battery energy in a homogeneous network; they are not identical in heterogeneous network. The cost of increasing the sensor's energy is much lower than the cost of having the same amount of energy with additional sensors. The effect of energy heterogeneity is indicated here. In addition to energy heterogeneity, there are two more types of heterogeneity: link heterogeneity [1] and computational heterogeneity [16]. These heterogeneities are essentially energy functions; energy heterogeneity is therefore the main heterogeneity. If no energy heterogeneity exists in a network, the link and computational heterogeneities will adversely affect the lifetime of the network.

An energy efficiency protocol that can have a major impact on network life and stability is one of the major problems of a WSN. [2]. Clustering can solve this problem because it is an effective approach that can efficiently use the network. This approach coordinates sensor nodes into clusters, each of which has a cluster head. The cluster heads can be fixed or motivated by themselves. In case of fixed cluster heads, the set of predefined heads is permanent throughout the entire process. If the self - motivated heads are used, the cluster heads are changed by means of a round robin approach.

We propose a heterogeneous multi - level network model that describes any finite level of heterogeneity. By considering the HNM implementation of our proposed model, we evaluate network performance. Accordingly, the HNM implementation (MLHNM) is called as MLHNM-1, MLHNM-2, MLHNM-3,..., MLHNM- $n$. The MLHNM-1 assumes that all sensor nodes in the WSN have the same amount of energy, i.e. a homogeneous network, used by the HNM protocol. The $n$-level heterogeneity of the 2-level, 3-level and 4-level assumes that nodes in a WSN have two, three, four and $n$ energy levels, respectively. In our work we demonstrate the heterogeneity of our model up to six levels. The original HNM protocol considers the residual energy and density of nodes in the cluster heads, which are two parameters. We consider the same cluster heads parameters for MLHNM.

The or

\section{RELATED WORKS}

The energy supply to nodes in a WSN is generally powered by battery, which is not rechargeable or replaceable. The major challenges in WSNs are improving the energy efficiency and maximizing the lifetime. In past years, several protocols have been discussed that mainly focus on the energy usage in order to prolong the network lifetime.

Heinzelman et al. [3] discuss low-energy adaptive clustering hierarchy $(\mathrm{LEACH})$ protocol based on clustering and it reduces the energy consumption so that the network lifetime increases [3]. It selects few nodes as the cluster heads dynamically established on their residual energy in round robin manner in the network. The cluster heads accumulate the data from the sensor nodes belonging to the clusters, aggregate it and then send the aggregated data to the base station.

The method in [4] discusses the LEACH-C, an enrichment over the LEACH protocol, by diffusing the cluster heads all over the network so that it can produce better performance. The method in [5] discusses the stable election protocol (SEP), an extension of LEACH, that considers the heterogeneous WSN with two-level of heterogeneity. It provides longer constancy region due to extra energy brought by more powerful sensor nodes; the heterogeneity cannot be extended for multi-level heterogeneous WSNs.

The method in [6] discusses the distributed energy efficient clustering (DEEC) protocol by considering 2-level and multilevel energy heterogeneity. In this protocol, for selecting the cluster 
heads, the probability is calculated based on the ratio of the residual energy of each node to the average energy of the network and the nodes with higher probability have more chance to be selected as cluster heads.

The study [7] discusses a method for increasing the lifetime by using ant colony optimization approach to prolong the lifetime of heterogeneous WSNs. In [8], the stochastic and equitable distributed energy-efficient clustering (SEDEEC) protocol is discussed for two-level heterogeneity. It selects the cluster heads based on the dynamic probability calculated by distributing the energy consumption uniformly in the whole network.

The paper [9] considers three types of nodes having different initial energy levels by assuming a single hop clustering topology and it analyzes the network lifetime.

The method in [10] discusses the energy efficient clustering and data aggregation (EECDA) protocol for the same heterogeneous network as given in [9]. It discusses a cluster head election technique and selects a path with maximum sum of residual energy for data transmission in place of the path with minimum energy consumption.

The method in [11] discusses distributed stable cluster head election (DSCHE) protocol to calculate the network lifetime and stability of a network by considering the similar heterogeneous WSN as in [9], [10]. It selects the cluster heads by using the weighted probability based on the ratio of the residual energy of each node to the average energy of the network.

The method in [12] discusses the balanced energy efficient network integrated super heterogeneous (BEENISH) protocol by considering four level of heterogeneity and it achieves longer stability, lifetime, and more effective messages than the DEEC and DDEEC.

In [13], the hybrid energy efficient distributed (HNM) clustering protocol is discussed that uses the residual energy as primary constraint and the node degree as the secondary constraint for cluster heads selection.

In method in [14] a heterogeneous HNM protocol is discussed for three level heterogeneous network model. It is an extension of the method in [13].

The method in [14] is further extended by the Singh et al. [15] that discusses an energy-efficient protocols using fuzzy logic for heterogeneous WSNs by considering five level of heterogeneity.

Kumar [17] discusses two distributed protocols namely, single-hop energy-efficient clustering protocol (S-EECP) and multi-hop energy-efficient clustering protocol (M-EECP). In SEECP, the cluster heads are elected by a weighted probability based on the ratio between residual energy of each node and average energy of the network. He observed that in single-hop communication where data packets are directly transmitted to the BS without any relay nodes, the nodes located far away from the BS have higher energy consumption because of long range transmission, and these nodes may die out first. This problem is solved in M-EECP by using multi-hop communication to the BS. M-EECP uses a greedy approach to solve the single source shortest problem to find the shortest path from each cluster head to the BS.

Farouk et al. [18] discuss a stable and energy-efficient clustering (SEEC) protocol and extend it to multi-level SEEC.

\section{NETWORK MODEL}

- Before the discussion of the network model, basic assumptions for WSN is listed.

- Initially all nodes have same capabilities, but different energies and are stationary after the deployment, each identified by a unique ID.

- The nature of nodes in the network is homogeneous or heterogeneous.

- Nodes are location-unaware, i.e. not equipped with a GPS capable antenna.

- The sensor nodes are left unattended after deployment, meaning thereby the battery recharging is not possible.

- There is only one stationary base station, in the center of the network area that has stable power supply; thus it has no energy, memory or computation restraints.

- Every node has the ability to perform data aggregation by which multiple data packets can be compressed into a single packet.

- The distance between sensor nodes is calculated on the basis of available received signal strength.

- Sensor nodes have the ability of regulating the transmission energy in accordance with the distance of the receiving nodes and the node failure is considered due to energy depletion.

- The energy consumption of data transmission from node $A$ to node $B$ is similar to the transmission from node $B$ to node $A$. Therefore, providing symmetric between wireless radio links.

- The sensor nodes equipped randomly in the monitoring area and nodes are maintained by the base station.

- The node IDs of the dead nodes are not reused for further processing.

\section{CLUSTERING AND DATA TRANSMISSION PROCESS}

Cluster formation is based on node energy levels. Two parameters are simultaneously considered during cluster formation, such as residual energy of the node and node density. The HNM protocol and its variants are based on a clustering approach that uses sensor node data correlation to form clusters in the information collection network. The clustering and transmission of data involves the following three phases: the formation of clusters and the selection of cluster heads, data collection and aggregation, and data transmission.

\subsection{CLUSTER FORMATION AND CLUSTER HEAD ELECTION}

We are using a simulated annealing during the cluster formation phase, used in the original HNM protocol, for the transmission of data to their respective cluster heads to minimise cluster nodes energy consumption. Just like in the HNM protocol, we select a few nodes at first, i.e. 5\%, as the cluster heads for the total nodes. This condition ensures that the cluster head doesn't fall into the sensor range of another cluster head [6]. Then the 
cluster heads are selected for the nodes with the highest probabilities. Following is the selection of the cluster head for MLHNM implementations.

In deciding on the cluster heads, we obtain the residual energy and node density of all nodes similar to the HNM protocol. A sensor node's residual energy and node density refers to the number of nodes within its sensing range. In order to circulate the load between the cluster heads, a node is attached to a head with a minimum degree. Once the cluster heads have been decided, each cluster head sends a broadcast message to all sensors as advertising. Each node decides its cluster head on the basis of the signal energy received and notifies the cluster head of its decision to correspond to the maximum signal energy that is received. The sensor nodes provide the cluster heads with a brief recognition about their choice. The clusters were therefore determined for the current round. Every head of the group produces and broadcasts a TDMA schedule to its members. Each cluster Member checks at the end of the election stage whether it has enough energy for the next round. If the energy of any cluster member goes to zero, the cluster is deleted and the remaining members are updated accordingly.

\subsection{DATA COLLECTION AND AGGREGATION}

The clusters collect the data from the monitoring region when they are formed and send it into their respective cluster heads. This process lasts several rounds until the energies of every node have not been depleted. The cluster heads aggregate the received data.

\subsection{TRANSMISSION OF DATA}

The cluster heads send the aggregated data to the base station during the data transmission phase. The clusters spend their energy on the collection and sending the data to their respective heads of clusters. This constitutes an iteration. You spend your energy on aggregating and sending the received data to the base station. The following iteration selects the cluster heads from the remaining nodes that have not yet been made in the current round. This process lasts several rounds until the energies of every node have not been depleted.

\subsection{ENERGY CONSUMPTION IN DIFFERENT PHASES}

In monitoring the area, the nodes in particular of cluster members use energy to collect the data for an activity and to forward the data to their respective cluster heads. They delete and send the redundancy to the base station; the two actions require energy. The study discusses the estimation of energy spent by WSN [3], [4].

\subsection{PSEUDOCODE OF THE PROPOSED PROTOCOL}

Step 1: Initially calculate the clusters of the sensor nodes deployed in the monitoring zone, their energy communication expenses and the initial probability for the cluster head (predefined).

Step 2: Find all the next sensors in the sensor range of the Cluster.
Step 3: Broadcast costs, e.g. energy levels information, sensor node residual energy, maximum energy and probability cluster heads for all neighboring sensor nodes.

Step 4: Set the probability of a sensor node to be elected as a cluster head.

Step 5: Initially, set the status of final cluster head as FALSE.

Step 6: After every clustering process time and network operating interval, repeat stage 7 - step 22 for clustering.

Step 7: If (selected cluster head is considered as a final cluster head, which is Null). The cluster head is selected based on a sensor with least cost.

Step 8: If (cluster head = node identity)

Step 9: If (Cluster Head Probability = 1). Broadcast the message from cluster head to its neighborhood cluster members with node identity, energy information and final cluster head.

Step 10: Set the status of final cluster head as TRUE

Step 11: Else

Step 12: Broadcast the message from cluster head to its neighborhood cluster members with node identity, energy information and final cluster head.

Step 13: Else If (Cluster Head Probability =1)

Step 14: Broadcast the message from cluster head to its neighborhood cluster members with node identity, energy information and final cluster head.

Step 15: Set the status of final cluster head as TRUE

Step 16: Else If set Cluster Head Probability as Random $(0,1)$

Step 17: Broadcast the message from cluster head to its neighborhood cluster members with node identity, energy information and final cluster head.

Step 18: Set probability of Cluster Head as previous Cluster Head probability

Step 19: Set the Probability of Cluster Head as min (Cluster Head Probability $\square$ 2, 1)

Step 20: While Previous Cluster Head probability = 1

Step 21: If (final cluster head $=$ FALSE)

Step 22: If (selected cluster head is a final cluster head $\neq$ Null)

Step 23: Then the final cluster head is considered to have least cost.

Step 24: Make final cluster head to join the cluster

Step 25: Maintain the identity of cluster head and identity of sensor nodes

Step 26: Else the message from cluster head is broadcasted to its neighborhood cluster members with node identity, energy information and final cluster head.

\section{EXPERIMENTAL EVALUATION}

In this section, we discuss the simulation results of the HNM protocol implementation for our proposed multilevel heterogeneity network model are discussed. We have already shown in Section 3 that our multilevel network model is capable to describe any level of heterogeneity. 
Table.1. Network lifetime in terms of number of alive nodes vs. number of rounds

\begin{tabular}{|c|c|c|c|c|c|c|}
\hline \multirow{2}{*}{$\begin{array}{c}\text { Alive } \\
\text { Sensor } \\
\text { Nodes }\end{array}$} & \multicolumn{7}{|c|}{ Number of Rounds } \\
\cline { 2 - 7 } & HNM-1 & $\begin{array}{c}\text { ML } \\
\text { HNM-2 }\end{array}$ & $\begin{array}{c}\text { ML } \\
\text { HNM-3 }\end{array}$ & $\begin{array}{c}\text { ML } \\
\text { HNM-4 }\end{array}$ & $\begin{array}{c}\text { ML } \\
\text { HNM-5 }\end{array}$ & $\begin{array}{c}\text { ML } \\
\text { HNM-6 }\end{array}$ \\
\hline 10 & 500 & 1242 & 1687 & 2166 & 2485 & 2987 \\
\hline 20 & 443 & 1200 & 1472 & 1750 & 2203 & 2597 \\
\hline 30 & 421 & 1096 & 1246 & 1596 & 1849 & 2259 \\
\hline 40 & 402 & 912 & 1120 & 1442 & 1645 & 2021 \\
\hline 50 & 375 & 748 & 994 & 1285.5 & 1491 & 1794.5 \\
\hline 60 & 350 & 645 & 891 & 1182 & 1388 & 1691 \\
\hline 70 & 329 & 547 & 793 & 1084 & 1290 & 1593 \\
\hline 80 & 302 & 441 & 687 & 978 & 1184 & 1487 \\
\hline 90 & 284 & 325 & 571 & 862 & 1068 & 1371 \\
\hline 100 & 241 & 254 & 301 & 405 & 497 & 602 \\
\hline
\end{tabular}

Alive Sensor Nodes

Fig.1. Network lifetime in terms of number of alive nodes vs. number of rounds

Table.2. Total energy dissipation vs. number of rounds

\begin{tabular}{|c|c|c|c|c|c|c|}
\hline \multirow{2}{*}{$\begin{array}{c}\text { No. } \\
\text { of } \\
\text { Rounds }\end{array}$} & \multicolumn{7}{|c|}{ Total Energy Dissipation } \\
\cline { 2 - 7 } & $\begin{array}{c}\text { HNL-1 } \\
\text { HNM-2 }\end{array}$ & $\begin{array}{c}\text { ML } \\
\text { HN-3 }\end{array}$ & $\begin{array}{c}\text { ML } \\
\text { HNM-4 }\end{array}$ & $\begin{array}{c}\text { ML } \\
\text { HN-5 }\end{array}$ & $\begin{array}{c}\text { ML } \\
\text { HNM-6 }\end{array}$ \\
\hline 0 & 36.5 & 35.5 & 32.41 & 28.1 & 25.54 & 20 \\
\hline 500 & 17.5 & 13.24 & 10.23 & 8.24 & 3.85 & 0 \\
\hline 1000 & 15 & 11.35 & 9.84 & 2.54 & 0 & 0 \\
\hline 1500 & 12 & 8.2 & 6.1 & 0 & 0 & 0 \\
\hline 2000 & 10 & 5 & 0 & 0 & 0 & 0 \\
\hline 2500 & 5 & 0 & 0 & 0 & 0 & 0 \\
\hline 3000 & 0 & 0 & 0 & 0 & 0 & 0 \\
\hline 3500 & 0 & 0 & 0 & 0 & 0 & 0 \\
\hline
\end{tabular}

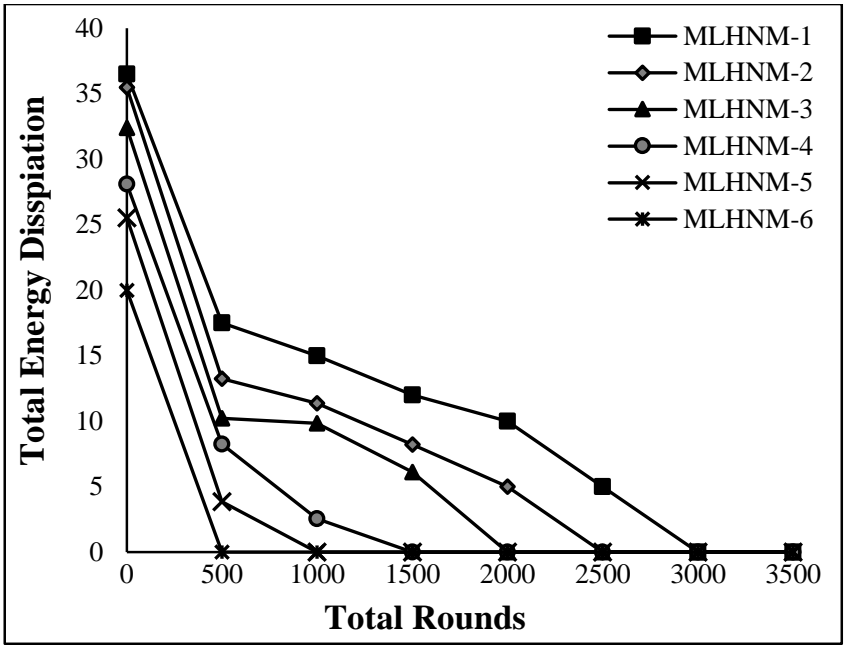

Fig.2. Total energy dissipation vs. number of rounds

Table.3. Aggregate delay vs number of sensor nodes

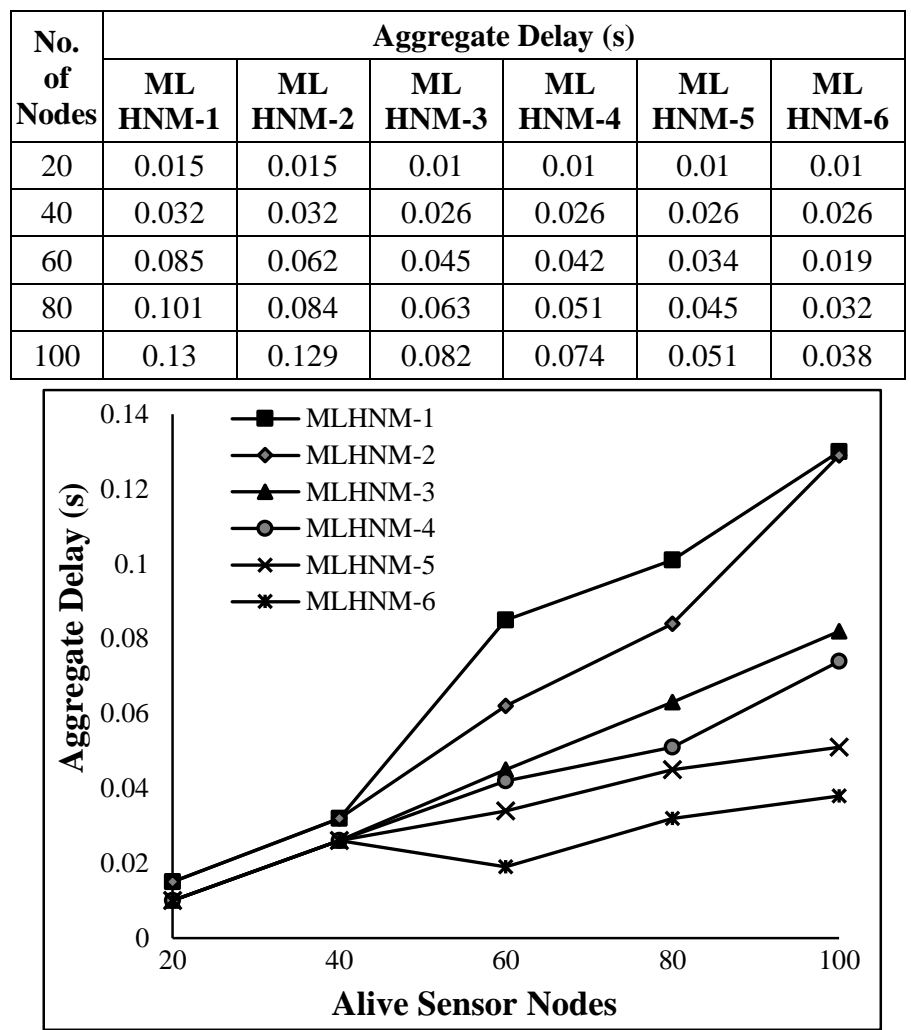

Fig.3. Aggregate delay vs number of sensor nodes

For simulation purpose, we have considered up to six level of heterogeneity that can have type-1, type-2, type- 3 , type- 4 , type- 5 , and type- 6 node in a WSN and accordingly we call the implementation of HNM as MLHNM-1, MLHNM-2, MLHNM3, MLHNM-4, MLHNM-5, and MLHNM-6. In original HNM protocol, the residual energy and node density have been used for calculating the probability of a sensor node to become a cluster head. Since our protocol MLHNM is based on the HNM, we use the same parameters for cluster head selection. In our simulations, we have used MATLAB by considering random deployment of 100 sensor nodes in a square field of dimension $100 \times 100 \mathrm{~m}^{2}$. 
Table.4. Throughput vs number of sensor nodes

\begin{tabular}{|c|c|c|c|c|c|c|}
\hline \multirow{2}{*}{$\begin{array}{c}\text { No. } \\
\text { of } \\
\text { Nodes }\end{array}$} & \multicolumn{7}{|c|}{ Throughput (Kbps) } \\
\cline { 2 - 7 } & HNM-1 & $\begin{array}{c}\text { ML } \\
\text { HNM-2 }\end{array}$ & $\begin{array}{c}\text { ML } \\
\text { HNM-3 }\end{array}$ & $\begin{array}{c}\text { ML } \\
\text { HNM-4 }\end{array}$ & $\begin{array}{c}\text { ML } \\
\text { HNM-5 }\end{array}$ & $\begin{array}{c}\text { ML } \\
\text { HNM-6 }\end{array}$ \\
\hline 20 & 0 & 0.12 & 0.52 & 0.74 & 1.3 & 2 \\
\hline 40 & 0.15 & 0.24 & 0.42 & 1.23 & 2.54 & 3.22 \\
\hline 60 & 2.8 & 5.2 & 7.85 & 10.36 & 13.52 & 16.21 \\
\hline 80 & 8.2 & 10.63 & 13.2 & 15.4 & 17.5 & 19.2 \\
\hline 100 & 10.6 & 17.2 & 15.6 & 18.2 & 20.6 & 22.5 \\
\hline
\end{tabular}

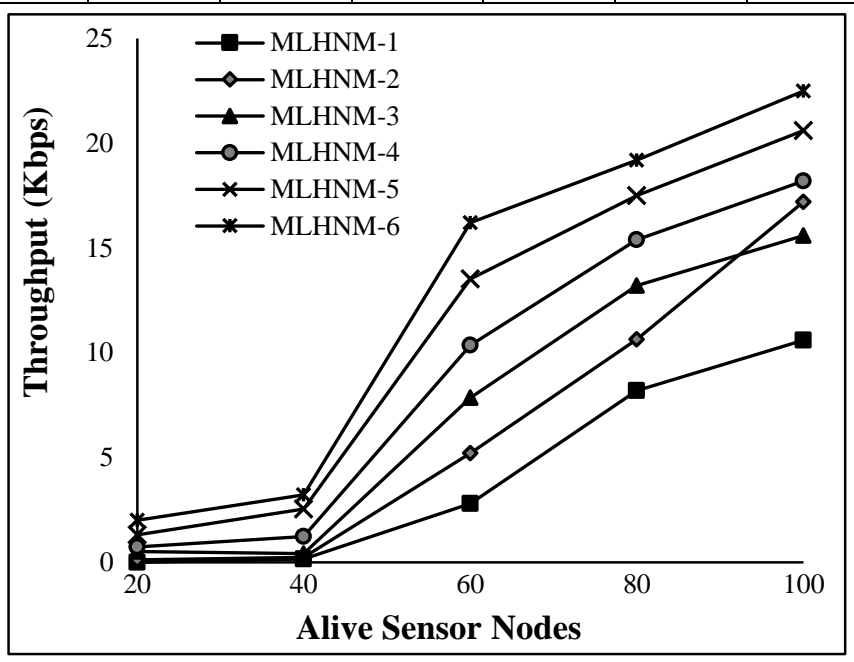

Fig.4. Throughput vs. number of sensor nodes

The Table. 1 and Fig. 1 shows the network lifetime in terms of number of alive nodes vs. number of rounds. The results show different variations for MLHNM-6 sustains for more rounds than the other methods. The Table. 2 and Fig. 2 shows the total energy dissipation vs. number of rounds. The result shows that the dissipation of energy is low in MLHNM-6 than other methods. The Table. 3 and Fig. 3 shows the aggregate delay vs number of sensor nodes. The result shows that the aggregate delay is lesser in MLHNM-6 than other methods. The Table.4 and Fig. 4 shows the throughput vs number of sensor nodes. The results show the throughput is high in MLHNM-6 than the other methods.

\section{CONCLUSIONS}

In this paper, we discussed a heterogeneous multilevel WSN network model that could describe any heterogeneity level at the end of this model. We have considered up to six heterogeneity levels in terms of energy for MLHNM implementation for the test validation, i.e. 1-level, 2-level, 3-level, 4-level, 5-level and 6-level heterogeneity. Increasing the level of heterogeneity extends network life to a considerable extent in comparison with the increase in network power. With six levels of heterogeneity, network life increases by $348.65 \%$, with the network energy growing by $84 \%$. The overall delay, throughput and traffic charges for all variants have also been calculated. The suggested HNM variants reduce over time and energy consumption significantly, as well as improve network performance and traffic loads.

\section{REFERENCES}

[1] I.F. Akyildiz, W. Su, Y. Sankarasubramaniam and E. Cayirci, "Wireless Sensor Networks: A Survey", Computer Networks, Vol. 38, No. 4, pp. 393-422, 2002.

[2] Tomi D. Raty, "Survey on Contemporary Remote Surveillance Systems for Public Safety", IEEE Transactions on Systems, Man, and Cybernetics, Part C (Applications and Reviews), Vol. 40, No. 5, pp. 493-515, 2010.

[3] W.R. Heinzelman, A.P. Chandrakasan and H. Balakrishnan, "Energy-Efficient Communication Protocol for Wireless Microsensor Networks", Proceedings of $33^{\text {rd }}$ Hawail International Conference on System Sciences, pp. 1-10, 2000.

[4] W.R. Heinzelman, A.P. Chandrakasan and H. Balakrishnan, "An Application Specific Protocol Architecture for Wireless Microsensor Networks", IEEE Transactions on Wireless Communications, Vol. 1, No. 4, pp. 660-670, 2002.

[5] G. Smaragdakis, I. Matta and A. Bestavros, "SEP: A Stable Election Protocol for Clustered Heterogeneous WSNs", Proceedings of $2^{\text {nd }}$ International Workshop on Sensor and Actor Network Protocols and Applications, pp. 1-11, 2004.

[6] L. Qing, Q. Zhu and M. Wang, "Design of A Distributed Energy-Efficient Clustering Algorithm for Heterogeneous WSNs", Computer Communications, Vol. 29, No. 12, pp. 2230-2237, 2006.

[7] Ying Lin, Jun Zhang, Henry Shu Hung Chung, Wai Hung Ip, Yun Li and Yu-Hui Shi, "An Ant Colony Optimization Approach for Maximizing the Lifetime of Heterogeneous Wireless Sensor Networks", IEEE Transactions on Systems, Man, and Cybernetics, Part C (Applications and Reviews), Vol. 42, No. 3, pp. 408-420, 2012.

[8] E. Brahim, S. Rachid and D. Aboutajdine, "Stochastic and Equitable Distributed Energy-Efficient Clustering (SEDEEC) for Heterogeneous Wireless Sensor Networks", International Journal of Ad Hoc and Ubiquitous Computing, Vol. 7, No. 1, pp. 4-11, 2011.

[9] D. Kumar, R.B. Patel and T.C. Aseri, "Prolonging Network Lifetime and Data Accumulation in Heterogeneous Sensor Networks", International Arab Journal of Information Technology, Vol. 7, No. 3, pp. 302-309, 2010.

[10] D. Kumar, R.B. Patel and T.C. Aseri, "EECDA: Energy Efficient Clustering and Data Aggregation Protocol for Heterogeneous Wireless Sensor Networks", International Journal of Computers Communications and Control, Vol. 6 , No. 1, pp. 113-124, 2011.

[11] D. Kumar, "Distributed Stable Cluster Head Election (DSCHE) Protocol for Heterogeneous Wireless Sensor Networks", International Journal of Information Technology, Communications and Convergence, Vol. 2, No. 1, pp. 90-103, 2012.

[12] T.N. Qureshi, N. Javaid, A.H. Khan, A. Iqbal, E. Akhtar and M. Ishfaq, "BEENISH: Balanced Energy Efficient Network Integrated Super Heterogeneous Protocol for Wireless Sensor Networks", Procedia Computer Science, Vol. 19, pp. 920-925, 2013.

[13] O. Younis and S. Fahmy, "Distributed Clustering in Ad-hoc Sensor Networks: A Hybrid, Energy-Efficient Approach", Proceedings of International Conference on Computer Communications, pp. 1-4, 2004. 
[14] S. Chand, S. Singh and B. Kumar, "Heterogeneous HNM Protocol for Wireless Sensor Networks", Wireless Personal Communications, Vol. 77, No. 3, pp. 2117-2139, 2014.

[15] S. Chand, S. Singh and B. Kumar, "Energy-Efficient protocols using Fuzzy Logic for Heterogeneous WSNs", Wireless Personal Communications, Vol. 86, No. 2, pp. 451475, 2015.

[16] Ali Sedighimanesh, Mohammad Sedighimanesh and Javad Baqeri, "Improving Wireless Sensor Network Lifetime using Layering in Hierarchical Routing", Proceedings of $2^{\text {nd }}$
International Conference on Knowledge-Based Engineering and Innovation, pp. 1145-1149, 2015.

[17] D. Kumar, "Performance Analysis of Energy Efficient Clustering Protocols for Maximising Lifetime of Wireless Sensor Networks", IET Wireless Sensor Systems, Vol. 4, No. 1, pp. 9-16, 2014

[18] F. Farouk, R. Rizk and F.W. Zaki, "Multi-Level Stable and Energy-Efficient Clustering Protocol in Heterogeneous Wireless Sensor Networks", IET Wireless Sensor Systems, Vol. 4, No. 4, pp. 159-169, 2014. 